\title{
Individuelle Mobilität hat Grenzen: \\ Luftreinhaltung (erst) durch Verkehrsverbote?
}

\author{
Nicolas Raschauer, Marco Dworschak und Magdalena Friedrich
}

Der Themenkomplex der Luftreinhaltung präsentiert sich - ungeachtet der aktuellen politisch brisanten Diskussionen - als juristische Querschnittsmaterie. Im öffentlichen Recht vorrangig im Bereich des Klimaschutz- und Umweltschutzrechts beheimatet, weisen staatliche Luftreinhaltemaßnahmen vielfach verkehrsrechtliche Anknüpfungspunkte auf bzw bedingen verkehrspolitische Lenkungs- und Planungsakte. Als solche werden nachfolgend sogenannte Verkehrs- oder Fahrverbote und -beschränkungen behandelt, die ein Spannungsfeld zwischen individueller Mobilität und Luftreinhaltung eröffnen.

Der vorliegende Beitrag widmet sich den rechtlichen Rahmenbedingungen und Voraussetzungen, unter denen Verkehrsverbote nach der gegebenen österreichischen Rechtslage grds zulässig sind.

Article note: Magdalena Friedrich und Marco Dworschak haben wesentliche Teile der im Manuskript verwerteten Quellen beschafft und ausgewertet. Das Manuskript wurde unter gleichnamigen Titel auch in JRP 2019, 27 ff. veröffentlicht. Der neuerliche Abdruck erfolgt mit freundlicher Genehmigung des Verlages Österreich und der Herausgeber der JRP.

\footnotetext{
N. Raschauer $(\square)$

HSSH Schaffhausen, Schaffhausen, Schweiz

E-Mail: nicolas.raschauer@hochschule-schaffhausen.ch

M. Dworschak · M. Friedrich

Lehrstuhl für Bank- und Finanzmarktrecht, Institut für Wirtschaftsrecht, Universität

Liechtenstein, Vaduz, Liechtenstein

E-Mail: marco.dworschak@uni.li; magdalena.friedrich@uni.li
}

S. Laimer, C. Perathoner (Hrsg.), Mobilitäts- und Transportrecht in Europa,

Bibliothek des Wirtschaftsrechts 2,

https://doi.org/10.1007/978-3-662-63635-0_7 


\section{Ausgangssituation}

Europas Ballungszentren ${ }^{1}$ sind mit ua durch den Kraftfahrzeugverkehr emittierten ${ }^{2}$ und im hohen Maße gesundheitsschädlichen Stickstoffoxiden ${ }^{3}$ belastet. ${ }^{4}$ Aufgrund der wiederholten Überschreitung ${ }^{5}$ europarechtlich vorgegebener Luftreinhaltegrenzwerte besteht hier besonderer Handlungsbedarf; dies trotz bereits in verschiedenen Mitgliedstaaten eingerichteter „Luftsanierungsgebiete“ “. ${ }^{6}$

Von einer breiten Öffentlichkeit wenig beachtet, erließ die Europäische Gemeinschaft seit den frühen 1980er-Jahren gesetzliche Regelungen, welche die Mitgliedstaaten zur Gewährleistung einer bestimmten Luftqualität verpflichten. ${ }^{7}$ Die Richtlinienvorgaben sind verursacherneutral ausgestaltet; dh sie stellen grundsätzlich nicht auf die Verursacher bestimmter Luftschadstoffe ab und geben somit von diesen unabhängig Immissionsgrenzwerte ${ }^{8}$ für bestimmte Luftschadstoffe wie zB Schwefeldioxid, Stickstoffoxide und Feinstäube vor. ${ }^{9}$ Diese Immissionsgrenzwerte basieren auf Empfehlungen und Studien der Weltgesundheitsorganisation $\mathrm{WHO}^{10}$

${ }^{1}$ Zwecks einfacheren Verständnisses wird hier exemplarisch auf die VO BGB1 II 2015/166 des BMLFUW über belastete Gebiete (Luft) zum UVP-G 2000 referenziert, zumal darin etwa die Ballungsgebiet Innsbruck und Salzburg als belastete Gebiete ausgewiesen werden.

${ }^{2}$ https://www.umweltbundesamt.de/daten/luft/luftschadstoff-emissionen-in-deutschland/stickstoffoxid-emissionen\#textpart-1, abgerufen am 30.1.2019.

${ }^{3}$ Näher https://www.umweltbundesamt.de/themen/stickstoffdioxid-belastung-hintergrund-zu-eu, abgerufen am 30.1.2019.

${ }^{4} \mathrm{Vgl}$ den aktuellen Bericht der Kommission: Erster Ausblick zur Entwicklung der Luftqualität, $\operatorname{COM}(2018) 446$ final/2.

${ }^{5}$ Selbst ein teilweise rückläufiger Trend bei der Immissionsbelastung - wie er etwa bzgl der $\mathrm{NO}_{2}$-Belastung teilweise beobachtet wird -, der indes noch nicht dazu führt, dass die Grenzwerte eingehalten werden, ist per se nicht geeignet, eine Vertragsverletzung gem Art 258 AEUV auszuräumen. So sprach der EuGH in C-488/15 (Kommission/Bulgarien), ECLI:EU:C:2017:67 Rz 69 aus, dass eine Überschreitung der einzuhaltenden Grenzwerte für sich genommen ausreicht, um eine Verletzung von Art 13 Abs 1 iVm Anhang XI Luftqualitätsrichtlinie festzustellen.

${ }^{6}$ Gemeint sind insbesondere die Grenzwerte der RL 2008/50/EG (nachfolgend Luftqualitätsrichtlinie) des Europäischen Parlaments und des Rates v 21.5.2008 über Luftqualität und saubere Luft für Europa (ABl [EU] $2008 \mathrm{~L}$ 152/1) für Stick(stoff)oxide $\left(\mathrm{NO}_{\mathrm{X}}\right)$.

Ferner jene für Stickstoffdioxid $\left(\mathrm{NO}_{2}\right)$, Schwefeldioxid $\left(\mathrm{SO}_{2}\right)$ und Feinstaubpartikel (PM), der RL 2001/81/EG des Europäischen Parlaments und des Rates v 23. 10. 2001 über nationale Emissionshöchstmengen für bestimmte Luftschadstoffe (ABl [EG] 2001 L 309/22) sowie entsprechende Grenzwerte der hieran anknüpfenden $\S \S 3$ ff IG-L (BGB1 I 1997/115 idgF), § 6 Emissionshöchstmengengesetz-Luft, BGB1 2003/34 sowie der darauf fussenden Luftreinhalteprogramme der Landeshauptleute ( $\S 9$ a ff IG-L).

${ }^{7}$ Will, Die Rechtsgrundlagen für Verkehrsverbote zur Einhaltung von $\mathrm{NO}_{2}$-Grenzwerten im Licht der aktuellen Rechtsprechung des BVerwG, NZV 2018, 393 (394).

${ }^{8}$ Immissionsgrenzwerte sind höchstzulässige, wirkungsbezogene Immissionsgrenzkonzentrationen, bei deren Unterschreitung nach den einschlägigen wissenschaftlichen Erkenntnissen keine schädigenden Wirkungen zu erwarten sind ( $\$ 2$ Abs 4 IG-L).

${ }^{9}$ Will, NZV 2018394.

${ }^{10} \mathrm{Vgl}$ ua WHO Regional Office for Europe Copenhagen in Regional Publications European Series, No. 91, Air Quality Guidelines for Europe ${ }^{2}$ (2000). 
und sind seit 2010 in der sog Luftqualitätsrichtlinie 2008/50/EG ${ }^{11}$ festgeschrieben. Sie gelten in „Gebieten und Ballungsräumen“, welche von den Mitgliedstaaten festzulegen sind. ${ }^{12}$ Diese Grenzwerte sind seit dem Ablauf des Übergangszeitraums seit dem 1. Jänner 2010 verbindlich. ${ }^{13}$

\section{Europäische Vorgaben}

Seit Jahren versuchen die Mitgliedstaaten vergeblich, den Herausforderungen der europäischen Luftqualitätsvorgaben gerecht zu werden. ${ }^{14}$

Nach Art 13 Abs 1 UAbs 2 iVm Anhang XI Luftqualitätsrichtlinie darf etwa die Konzentration von Stickstoffdioxid einen Jahresmittelwert von 40 Mikrogramm pro $\mathrm{m}^{3}$ Luft $^{15}$ nicht überschreiten; ${ }^{16}$ zudem definiert die Luftqualitätsrichtlinie eine Schwelle von 50 Mikrogramm, die an nicht mehr als 35 Tagen im Jahr überschritten werden darf. ${ }^{17}$

Aus Art 13 Abs 1 Luftqualitätsrichtlinie ergibt sich keine Pflicht zur Anordnung bestimmter Maßnahmen, welche die Einhaltung der einschlägigen Luftgrenzwerte gewährleisten, sondern beschränkt sich auf eine „Ergebnisverpflichtung“. ${ }^{18}$ Den Mitgliedstaaten bleibt zunächst die Wahl überlassen, welche Maßnahmen sie zur Verwirklichung von Art 13 Abs 1 Luftqualitätsrichtlinie setzen: Als Beispiele seien hier etwa die Einführung einer „City-Maut“ gem $\S 8$ Abs 5 F-VG, die Statuierung von Geschwindigkeitsbeschränkungen, den Ausbau des öffentlichen Verkehrs, die Erhöhung von Verkehrssteuern (etwa im Anwendungsbereich des Mineralölsteuergesetzes), die Gewährung von Förderungen, aber auch die Einführung von

\footnotetext{
${ }^{11} \mathrm{Vgl} \mathrm{FN} 6$.

${ }^{12}$,[Sanierungs]Gebiete“ sind gem Art 2 Nr 16 Luftqualitätsrichtlinie iVm § 2 Abs 8 IG-L Teile des Hoheitsgebiets eines Mitgliedstaats, die dieser Mitgliedstaat für die Beurteilung und Kontrolle der Luftqualität abgegrenzt hat. „Ballungsräume“ sind gem Art $2 \mathrm{Nr} 17$ Luftqualitätsrichtlinie städtische Gebiete mit einer Bevölkerung von mehr als 250.000 Einwohnern oder, falls 250.000 oder weniger Einwohner in dem Gebiet wohnen, Gebiete mit einer Bevölkerungsdichte pro km², die von den Mitgliedstaaten festzulegen ist.

${ }^{13}$ Der EuGH hat iZm Art 13 Abs 1 UAbs 2 Luftqualitätsrichtlinie, wonach die Grenzwerte von dem dort festgelegten Zeitpunkt an „,nicht mehr überschritten werden [dürfen]“, von einer „Ergebnisverpflichtung" der Mitgliedstaaten gesprochen; vgl EuGH 28.11.2013, C-404/13 (Client Earth), ECLI:EU:2014:2382 Rz 30.

${ }^{14}$ Die EU-Kommission leitete zB im Mai 2018 gegen Frankreich, Großbritannien, Italien, Ungarn, Rumänien und Deutschland Vertragsverletzungsverfahren wegen Verstoßes gegen die Luftqualitätsrichtlinie ein (für einen Überblick vgl COM [2018] 446 final/2). In Deutschland überschritten 201766 Städte die Stickoxidgrenzwerte, vgl Graf/Schwischei, Wer legt Grenzwerte für Luftschadstoffe fest?, Salzburger Nachrichten vom 29.1.2019.

${ }^{15}$ „Luft“ ist gem Art 2 Nr 1 Luftqualitätsrichtlinie iVm $§ 2$ Abs 6a IG-L die Außenluft in der Troposphäre mit Ausnahme von bestimmten Arbeitsstätten.

${ }^{16}$ Das IG-L schreibt für Stickstoffdioxid einen Grenzwert von 30 Mikrogramm pro $\mathrm{m}^{3}$ Luft vor.

${ }^{17}$ Ende 2019 sind die Grenzwerte wieder überprüft worden.

${ }^{18}$ Will, NZV 2018, $394 \mathrm{f}$.
} 
sogenannten Fahrverboten und Verkehrsbeschränkungen gem $\S 43$ Abs 2a StVO oder $\S \S 9 \mathrm{a}$ ff iVm $\S \S 10 ; 14$ IG-L ${ }^{19,20}$ genannt.

Verstößt ein Mitgliedstaat gegen die Zielvorgaben der Luftqualitätsrichtlinie und werden die darin festgelegten verbindlichen Grenzwerte überschritten, legt die Richtlinie dem Mitgliedstaat normative „Daumenschrauben“ an, ${ }^{21}$ als solche dienen sodann die sogenannten Luftqualitätspläne iSd Art 23 Luftqualitätsrichtlinie bzw $\S \S 9 \mathrm{a}$ ff IG-L:

Diese Luftqualitätspläne müssen gem Art 23 Abs 1 UAbs 2 Satz 1 Luftqualitätsrichtlinie ,geeignete Maßnahmen“ enthalten, welche effektiv gewährleisten, dass die Grenzwerte in ,,absehbarer Zeit“ eingehalten werden. Der Zeitraum der (weiteren) Nichteinhaltung muss dabei „,so kurz wie möglich gehalten werden““.22

Hinsichtlich der Interpretation dieser Bestimmung vertritt das dt BVerwG die Auffassung, dass die zu ergreifenden Maßnahmen jedenfalls nicht unmittelbar zur Zielerreichung führen müssen; vielmehr sei nach Maßgabe des Verhältnismäßigkeitsgrundsatzes ein Vorgehen in mehreren Stufen zur korrekten Anwendung der Vorgaben der Luftqualitätsrichtlinie ausreichend. ${ }^{23}$

Im Unterschied dazu hat sich der EuGH bislang noch nicht eindeutig positioniert; in seiner Entscheidung C-336/17 stellte der Gerichtshof lediglich fest, dass Maßnahmen, die erst zwischen 2020 und 2024 (also zehn oder sogar erst 14 Jahre nach der erstmaligen Feststellung einer Grenzwertüberschreitung) wirksam werden, nicht den Vorgaben der Luftqualitätsrichtlinie entsprechen würden. ${ }^{24}$

Wie nun rezente Entscheidungen des dt $\mathrm{BVerwG}^{25}$ gezeigt haben, kommt im Fall von Grenzwertüberschreitungen als sofort ,wirksame Maßnahme“ ${ }^{26}$ derzeit insb ein

\footnotetext{
${ }^{19} \mathrm{Ob}$ und inwieweit Fahrverbot auf die eine oder andere gesetzliche Grundlage gestützt werden können, bzw wie sich die einzelnen Rechtsgrundlagen zueinander verhalten, ist an dieser Stelle nicht zu untersuchen (vgl dazu etwa Muzak, Verkehrsbeschränkungen zwischen IG-L und StVO, ZVR 12a (2015), 475 [475 ff]). Zu kompetenzrechtlichen Abgrenzungsfragen siehe nur A. Hauer, Verfassungsfragen pauschaler Geschwindigkeitsbeschränkungen nach dem IG-L, ZVR 12a (2015), 440 (440 ff).

${ }^{20}$ Die oft ungenau als „Fahrverbote“ bezeichneten Verkehrsverbote und -beschränkungen für Fahrzeuge mit Verbrennungsmotoren, va für solche mit Dieselmotoren, zur Einhaltung insbesondere von Stickstoffdioxid-Grenzwerten bilden das aktuelle umweltpolitische Thema in der Öffentlichkeit.

${ }^{21}$ Will, NZV 2018, 395.

${ }^{22}$ Beye, Anordnung von Verkehrsverboten für Diesel-Kraftfahrzeuge, ZJS 2018/6 528, (533).

${ }^{23}$ Dt BVerwG 27.2.2018, 7 C 30.17 Rz 59; vgl auch Will, NZV 2018, 395.

${ }^{24}$ EuGH 22.2.2018, C-336/16 (Kommission/Polen), ECLI:EU:2018:94 Rz 99.

${ }^{25}$ Siehe dt BVerwG 27.2.2018, 7 C 3017 betreffend ein Verkehrsverbot für Dieselfahrzeuge in der Umweltzone Stuttgart und dt BVerwG 27.02.2018, 7 C 26.16 betreffend (beschränkte) Verkehrsverbote für (bestimmte) Dieselfahrzeuge - Luftreinhalteplan Düsseldorf; vgl Beye, ZJS 2018, $528 \mathrm{f}$.

${ }^{26}$ Dies unter der Annahme, dass sich Verkehrsverbote für bestimmte Kfz mit Verbrennungsmotoren als am besten geeignete Maßnahmen zur schnellstmöglichen Einhaltung der überschrittenen Grenzwerte erweisen und ex ante keine vergleichbar effektiven Alternativmaßnahmen zur Verfügung stehen. Diesfalls müssen diese Verbote im Licht des effet-utile-Gedanken und des Loyalitätsprinzips des Unionsrechts grds angeordnet werden (vgl auch dt BVerwG 27.2.2018, 7 C 30/17, NVwZ
} 
(zumindest) partielles Fahrverbot für motorbetriebene, vor allem dieselbetriebene Fahrzeuge, ${ }^{27}$ in Betracht. Diese Maßnahme wäre als Bestandteil eines solchen Luftreinhalteplans gem Art 23 Abs 1 Luftqualitätsrichtlinie umzusetzen.

Mit Blick auf die österreichische Rechtslage stünde ein solches Verbot auf den ersten Blick in diametralem Widerspruch zu § 1 StVO, wonach öffentliche Straßen von jedermann unter den gleichen Bedingungen benützt werden dürfen. Der Gemeingebrauchsregel der StVO kommt jedoch kein grundrechtlicher Charakter zu; sie ist folglich nicht als absolut zu sehen. Sie überlässt den zuständigen Vollziehungsbehörden Spielraum ${ }^{28}$ und steht der Erlassung von Fahrverboten nicht entgegen.

Nachdem die gesetzliche Zulässigkeit von Fahrverboten bejaht werden kann, stellt sich somit die Frage, wie nun mit einem solchen Fahrverbot in Österreich umzugehen wäre? Im Anschluss werden am Beispiel des IG-L die Vorgaben und Grenzen für die Einführung von Verkehrsbeschränkungen skizziert.

\section{Ausgestaltung von Fahrverboten am Beispiel IG-L}

Bereits nach geltendem Recht ${ }^{29}$ können Fahrverbote in belasteten Ballungsräumen, in denen Grenzwerte überschritten werden, mittels Verordnung oder Bescheid angeordnet werden. Personen, die von einer Grenzwertüberschreitung unmittelbar betroffen sind, sowie anerkannte Umweltorganisationen gem § 19 Abs 6 ff UVP-G, haben einen Rechtsanspruch auf Erlassung entsprechender Maßnahmen ( $\$$ a Abs 11 IG-L). ${ }^{30}$

Zuständig für die Erlassung eines solchen Fahrverbots ist der jeweilige Landeshauptmann, welcher jedoch vor Erlassung eines solchen eine Grundlagenforschung $^{31}$ vorzunehmen und zwingend folgende (Planungs-)Grundsätze zu berücksichtigen hat:

\footnotetext{
2018, $883 \mathrm{Rz} 35)$.

${ }^{27}$ Nach Angaben des dt Umweltbundesamtes (FN 2) sind Emissionen von dieselbetriebenen Pkw die Hauptquelle für Stickoxid in Ballungsräumen (der Verkehrsbereich trägt zu ca $60 \%$ an der Gesamt- $\mathrm{NO}_{2}$-Belastung in Deutschland bei; davon wiederum gehen 72,5 \% der Immissionen auf Diesel-Pkw).

${ }^{28} \mathrm{Vgl}$ allgemein OGH RS0111848 ua.

${ }^{29} \mathrm{Vgl} \S 10 \mathrm{iVm} \S 14$ IG-L.

${ }^{30} \mathrm{Vgl}$ bereits EuGH 25.7.2008, C-237/07 (Janecek), ECLI:EU:C:2008:447; VwGH 19.2.2018, Ra 2015/07/0074. Nach § 9a Abs 11 IG-L ist im Zweifel über das Bestehen eines Anspruches mit Bescheid abzusprechen.

${ }^{31}$ Nach ständiger Rechtsprechung des VfGH zum Raumplanungsrecht (vgl VfSlg 8280/1978, 19.890/2014 ua) kommt den Vorschriften des Raumplanungsrechts über die Erarbeitung der Entscheidungsgrundlagen für rechtsverbindliche Planungen besondere Bedeutung zu; dieser Grundsatz kann auf gegenständliche Verordnungen gem IG-L übertragen werden. Der VfGH hat in solchen Fällen im Verordnungsprüfungsverfahren nach Art. 139 B-VG zu prüfen, ob der Verordnungsgeber die im Gesetz zur Gewinnung einer ausreichenden Entscheidungsgrundlage
} 
1. Zunächst ist zu klären, welche Schutzgüter (bspw die Gesundheit der Anrainer) durch Luftschadstoffe beeinträchtigt werden; dem ist sodann im Sinne des Verursacherprinzips vorzubeugen. Nach Möglichkeit sind Luftschadstoffe an ihrem Ursprung zu bekämpfen.

2. Zudem sind Emittenten, die im Beurteilungszeitraum einen nennenswerten Einfluss auf die Immissionsbelastung, insbesondere im Zeitraum der Überschreitung des Immissionsgrenzwerts, gehabt haben, zu berücksichtigen;

3. Sodann sind die Maßnahmen vornehmlich bei diesen hauptverursachenden Emittenten unter Berücksichtigung der auf sie fallenden Anteile an der Immissionsbelastung, des Reduktionspotenzials und des erforderlichen Zeitraums für das Wirksamwerden der Maßnahmen zu setzen; dabei sind vorrangig solche Maßnahmen anzuordnen, bei denen den Kosten der Maßnahme eine möglichst große Verringerung der Immissionsbelastung gegenübersteht;

4. Solche Maßnahmen sind nicht vorzuschreiben, wenn der mit der Erfüllung der Maßnahmen verbundene Aufwand außer Verhältnis zu dem mit den Anordnungen angestrebten Erfolg steht;

5. Mit solchen Maßnahmen verbundene Eingriffe in bestehende Rechte sind auf das unbedingt erforderliche Maß zu beschränken; bei der Auswahl von Maßnahmen sind die jeweils gelindesten, zum Ziel führenden Mittel zu ergreifen;

6. Außerdem ist neben der Höhe der Immissionsbelastung auf die Häufigkeit der Grenzwertüberschreitungen, die zu erwartende Entwicklung der Emissionen des betreffenden Luftschadstoffs sowie auf eingeleitete Verfahren, angeordnete Sanierungsmaßnahmen und gebietsbezogene Maßnahmen nach IG-L Bedacht zu nehmen. Darüber hinaus sind andere Verwaltungsvorschriften, sofern diese Einfluss auf die Immissionssituation haben, zu berücksichtigen;

7. Ferner sind sonstige öffentliche Interessen (Aufrechterhaltung der Gesundheitsversorgung, die Versorgung der Bevölkerung etc) einzubeziehen.

\section{Interessenabwägung als Dreh- und Angelpunkt der Fahrverbotserlassung}

Das Unionsrecht (Art 20; 52 Abs 1 GRC) und das nationale Verfassungsrecht (vgl etwa Art 7 Abs 1 B-VG) verpflichten die zuständigen nationalen Behörden zu einer Interessenabwägung, bevor Fahrverbote erlassen werden können. § 9b Z 5 IG-L, der diese Vorgabe der Interessenabwägung konkretisiert, ist dementsprechend unionsrechts- und verfassungskonform anzuwenden und somit Dreh- und Angelpunkt der

vorgesehene Vorgangsweise eingehalten hat. Insbesondere zur Durchsetzung der gesetzlich definierten (Planungs)ziele ist die Durchführung einer Grundlagenforschung - unabhängig davon, ob sie vom Gesetzgeber ausdrücklich vorgesehen ist oder nicht - unabdingbar (vgl zB VfSlg 19.126/2010, 19.760/2013 uva). Diese Grundlagenforschung hat in allgemeinen Überlegungen zu bestehen, welche die Grundlage für die jeweilige (Planungs)entscheidung bilden und als solche auch erkennbar und nachvollziehbar sind (zB VfSlg 14.537/1996, 19.075/2010). 
unions- und verfassungsrechtlich vorgegebenen Interessenabwägung: Die zuständige Behörde hat vor der Erlassung eines Fahrverbotes die Rechte von Anrainern, welche von den Grenzwertüberschreitungen im relevanten Ballungsgebiet unmittelbar betroffen sind (ua auf Gesundheit und Leben gem Art 2 und 8 EMRK), mit jenen von betroffenen Fahrzeugeigentümern und -haltern, die ihrerseits von einem Fahrverbot gem Art 1 1. ZP EMRK in ihrem Schutz auf Eigentum unmittelbar betroffen wären, abzuwägen. Bei einer solchen Interessenabwägung hat die zuständige Behörde auch zu berücksichtigen, inwieweit die Bevölkerung und die Wirtschaft im relevanten Ballungsgebiet weiterhin adäquat versorgt werden können.

\section{Arten von Fahrverboten}

Aus dem Gesagten folgt schließlich, dass sich Fahrverbote primär auf dieselbetriebene Fahrzeuge als relevante Verursacher konzentrieren müssen. ${ }^{32}$ Wie den bereits zitierten Entscheidungen des dt BVerwG entnommen werden kann, sind verschiedene Arten von Fahrverboten denkbar: Einerseits kommen streckenbezogene (zB für innerstädtische Hauptverkehrsstraßen), andererseits zonale Verkehrsverbote man denke an die autofreie Kärntner Straße in Wien - zur Einhaltung der Luftreinhaltegrenzwerte in Betracht

Des Weiteren wäre es auch denkbar, Fahrverbote zeitlich gestuft einzuführen: In einer ersten Stufe könnte ,ab sofort“ ein Fahrverbot für ältere Fahrzeuge, konkret Dieselfahrzeuge bis zur Abgasnorm Euro 0-4 und Fahrzeuge mit Ottomotoren unterhalb der Abgasnorm Euro-3, eingeführt werden, während zonale Verbote für Euro-5-Fahrzeuge erst ab einem späteren Zeitpunkt vorgesehen werden könnten. ${ }^{33}$ Sollte dies nicht ausreichen, Grenzwertüberschreitungen hintanzuhalten, könnten Verkehrsverbote entgegen dem von dt BVerwG entwickelten Ansatz auch „sofort“ für Euro-6-Fahrzeuge verordnet werden. Dies vor allem, wenn man bedenkt, dass Euro 6-Fahrzeuge die EU-rechtlich definierten Grenzwerte aktuell um $100 \%$ überschreiten. ${ }^{34}$

Aus dem Verhältnismäßigkeitsgrundsatz iVm § 9b IG-L ergibt sich schlussendlich, dass Verkehrsverbote nicht ,pauschal“ verordnet werden dürfen - gebotenenfalls sind Ausnahmen, etwa für Elektrofahrzeuge, in der Fahrverbotszone wohnende Anrainer, oder Unternehmen, die bspw zu einer Baustelle anfahren müssen, zu berücksichtigen. Ausnahmen sind daher dann zu verordnen, wenn diese Ausnahme zur Vermeidung von „Härtefällen“ erforderlich ist (dies betrifft sowohl zonale als auch streckenbezogene Fahrverbote). ${ }^{35}$ Allerdings sind im Zweifel die Ausnahmeklauseln

\footnotetext{
${ }^{32} \mathrm{Vgl}$ Beye, ZJS 2018, $534 \mathrm{f}$.

${ }^{33}$ Beye, aaO mVa dt. BVerwG 27. 2. 2018, 7 C 26.16, Rz 39 f. und 7 C 30.17, Rz 42 f.

${ }^{34}$ Faßbender, Der Dieselskandal und der Gesundheitsschutz, NVwZ2017, $1995(1996,1998)$ mwN.

${ }^{35} \mathrm{Vgl}$ die Ausführungen von Will, NZV 2018, 399, 402, zur ähnlich gelagerten Argumentation in Deutschland.
} 
nach Art des $§ 14$ Abs 2 IG-L im Sinne der ratio der Luftqualitätsrichtlinie restriktiv anzuwenden und auszulegen.

Hier ist zudem darauf hinzuweisen, dass gegen die Zulässigkeit von Fahrverboten (va auf verfassungsrechtlicher Ebene) nicht eingewendet werden kann, dass entsprechende (va streckenbezogene) Maßnahmen zu Verkehrsverlagerungen führen könnten. Dass das zu erwartende Ausweichverhalten von Anrainern oder die längere Fahrtstrecke von Arbeitnehmern, bspw als Folge von Fahrverboten, andernorts sogar zu einer Zunahme von Emissionen führen könnte, muss nach der Konzeption von IG-L und Luftqualitätsrichtlinie nicht beanstandet werden. Es spricht im Licht der Systematik und des Telos der genannten Rechtsquellen gerade nicht gegen die Erlassung von Verkehrsverboten. Weder die Luftqualitätsrichtlinie noch das IG-L statuieren ein allgemeines Minimierungsgebot für einzelne lokale Gebiete, die von einem Fahrverbot betroffen sein könnten, sondern lediglich die Einhaltung der Immissionsgrenzwerte in bestimmten Gebieten und Ballungsräumen. ${ }^{36}$ Anderes gilt erst, wenn es durch ein Fahrverbot zu Umlenkung von Verkehrsströmen kommt, die wiederum zur Überschreitung von Grenzwerten an anderer Stelle führen; dies kann - iS einer ultima ratio - gegen die Erlassung von Fahrverboten sprechen.

Bei unions- und gesetzeskonformer Planung können solche „Extremeffekte“ indes, eine vorausschauende behördliche Planung vorausgesetzt, in den Luftreinhalteplänen vorab berücksichtigt und damit abgefedert werden.

\section{Resümee}

Was folgt aus dem Gesagten für Österreich? Droht Österreich ebenfalls eine Lawine an Fahrverboten? Die Antwort ist: Nein; das wäre ein voreiliger und überschießender Befund. ${ }^{37}$

Österreich erhielt vor Jahren zwar ebenfalls einen „Mahnbrief“ der Kommission; verschiedene Vertragsverletzungsverfahren wurden eingeleitet und sind noch anhängig. Mittlerweile sind die Grenzwerte aber im Rahmen. Nach den jüngsten veröffentlichten Zahlen hat sich die Luftqualität in österreichischen und europäischen Ballungszentren verbessert. ${ }^{38}$ Gegen die Erlassung von Fahrverboten in österreichischen Städten sprächen daher derzeit die voranstehend dargelegten VerhältnismäBigkeitserwägungen, die freilich einzelfallbezogen vorzunehmen wären.

Was bleibt, ist ein letztlich akademischer Befund: Es ist positiv, dass durch die gerichtliche Prüfung von Fahrverboten eines der letzten umweltrechtlichen „Tabus“ gebrochen wurde. Dank der Pionierleistung der deutschen Rechtsprechung hat auch in Deutschland das deutliche Bekenntnis zur Luftqualitätsrichtlinie und zu umfassendem Gesundheits- und Umweltschutz die politische und juristische Diskussion beflügelt.

\footnotetext{
${ }^{36} \mathrm{Vgl}$ Will, NZV 2018, $404 \mathrm{f}$.

${ }^{37} \mathrm{Vgl}$ den aktuellen Kommissionsbericht COM(2018) 446 final/2 (FN 4).

${ }^{38}$ Graf/Schwischei, Grenzwerte; Kommissionsbericht COM(2018) 446 final/2 (FN 4).
} 
Es bleibt im Ergebnis zu hoffen, dass im Umweltrecht keine weiteren beschwerlichen „Reisen“ mehr bis nach Luxemburg beschritten werden müssen, bis die Mitgliedstaaten ihre europäischen Verpflichtungen vollständig erfüllen.

Open Access Dieses Kapitel wird unter der Creative Commons Namensnennung 4.0 International Lizenz (http://creativecommons.org/licenses/by/4.0/deed.de) veröffentlicht, welche die Nutzung, Vervielfältigung, Bearbeitung, Verbreitung und Wiedergabe in jeglichem Medium und Format erlaubt, sofern Sie den/die ursprünglichen Autor(en) und die Quelle ordnungsgemäß nennen, einen Link zur Creative Commons Lizenz beifügen und angeben, ob Änderungen vorgenommen wurden.

Die in diesem Kapitel enthaltenen Bilder und sonstiges Drittmaterial unterliegen ebenfalls der genannten Creative Commons Lizenz, sofern sich aus der Abbildungslegende nichts anderes ergibt. Sofern das betreffende Material nicht unter der genannten Creative Commons Lizenz steht und die betreffende Handlung nicht nach gesetzlichen Vorschriften erlaubt ist, ist für die oben aufgeführten Weiterverwendungen des Materials die Einwilligung des jeweiligen Rechteinhabers einzuholen.



